\title{
An Analytical Method for Coaxial Helicopter Ground Resonance
}

\author{
Xiangyi Liü, ${ }^{1, a}$, Shuyan $\mathrm{Liu}^{1}$, Jing $\mathrm{Wu}^{1}$, Shansong Song ${ }^{1}$ and Guocai $\mathrm{Hu}^{2}$ \\ ${ }^{1}$ School of Basic Sciences for Aviation, Naval Aviation University, Yantai, China \\ ${ }^{2}$ Department of Aviation Technology, Nanshan Aeronautical College, Yantai, China
}

\begin{abstract}
A time-frequency analytical method is presented to analyze physical mechanism of coaxial helicopter ground resonance. Eigenvalue calculation and numerical integration of disturbance equations of motions are used to obtain modal characters and time-domain response characters of coaxial helicopter ground resonance, and the interaction between rotors and body is revealed according to response of various DOFs. The analysis results show that regressive lag mode with upper rotor character is the most instability mode. In dynamic instability region, coaxial helicopter ground resonance is mainly due to energy transferred between periodic lag motion of upper rotor and body roll rotation. For this instability mode, energy transferred between periodic lag motion of lower rotor and body roll rotation is also existed, and it can enhance ground resonance instability of coaxial helicopter.
\end{abstract}

\section{Introduction}

Helicopter ground resonance is one of dynamics problems must be solved in model design. For a single rotor helicopter with tail, the domestic and foreign scholars have done a lot of research about ground resonance, from Investigation analysis for ground resonance $^{[1,2]}$, the influence of the design parameters ${ }^{[3-5]}$, nonlinear analysis of the helicopter ground resonance ${ }^{[6-8]}$, active or passive control ${ }^{[9,10]}$ and ground resonance test verification $^{[11,12]}$. Many well-known conclusions have been made and the problem of ground resonance in model development has been well solved ${ }^{[13-16]}$. Coaxial helicopter compared with single rotor helicopter, the most significant difference is that body is equipped with reverse rotation of upper/lower rotor. In the published literature, there are few papers on physical investigation of coaxial helicopter ground resonance. We find that there is no systematic analysis and explanation about the influence of physical investigation and design parameters of upper/lower rotor in ground resonance, therefore, this poses potential uncertainties in the design analysis、 use and maintenance of coaxial helicopter.

Referencing to [2], rotor periodic lag motion、 body pitch motion and body roll motion is expressed as rotation DOFs in complex coordinates, aiming at the analytical model for single rotor helicopter ground resonance without structural damping, and physical investigation of single rotor helicopter ground resonance is revealed according to the intrinsic relation between complex eigenvector and rotor-fuselage mutual stimulate, and applies this method to analysis for a helicopter ground resonance ${ }^{[17]}$. Coaxial helicopter has two reverse rotation rotors (upper rotor and lower rotor), adding the impact of rotor and fuselage structure damping, so the relationship of various modes of eigenvectors become complex, and increasing physical analytical difficulty ${ }^{[18]}$. Because the damping that endangers the most unstable mode of helicopter ground resonance is minimal, and numerical integration of disturbance equations of motions are used to obtain modal response, at the same time it is not difficult to obtain process data of speed and acceleration of various DOFs, then we can analyze energy transfer relationship of various DOFs. Therefore, this paper presents a time-frequency analytical method for the coaxial articulated rotor helicopter to analyze the interaction between lag (upper rotor and lower rotor) and body motion, and revealing the physical investigation of coaxial helicopter ground resonance.

\section{Analytical model}

Each rotor(upper rotor and lower rotor) of coaxial helicopter has $N$ blades, with the reverse rotation speed of $\Omega$, as shown in Figure 1. When looking down, the lower rotor rotates counterclockwise, the hub coordinate system of lower rotor is $o_{1} x_{1} y_{1} z_{1}$, and following the hub movement, the origin $o_{1}$ is located at the center of the hub of lower rotor, $o_{1} x_{1}$ points to the tail of the fuselage; Upper rotor rotates clockwise as viewed from above, Upper rotor hub coordinate system is $o_{2} x_{2} y_{2} z_{2}$, and the origin $o_{2}$ is located at the center of the hub of upper rotor, $\mathrm{O}_{2} x_{2}$ also points to the tail of the fuselage; All lag hinge extension is $e$, and lag angles of blades are $\zeta_{1} 、 \zeta_{2}$, azimuth angles of the blades are $\psi_{1}$ and $\psi_{2}$, respectively; The fixed coordinate system is oxyz, which coincides with the body coordinate system at rest. Assuming that the center of hub and body rotate around the

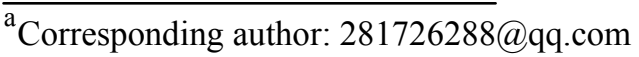


instantaneous center, roll angle around $x$-axis is $\varphi_{x}$, the instantaneous center is located at $h_{x}$ below the center of hub of lower rotor, and elevation angle around $y$-axis is $\varphi_{y}$, and the instantaneous center is located at $h_{y}$ below center of hub of lower rotor, the distance between upper rotor and lower rotor is $h$. For convenience, regarding the instantaneous center as the origin of coordinates, For the model of helicopter testing in wind tunnel, the instantaneous center is located at the universal hinge off uselage (adjust the center of mass and universal hinge coincidence), this time, $h_{x}=h_{y}$.
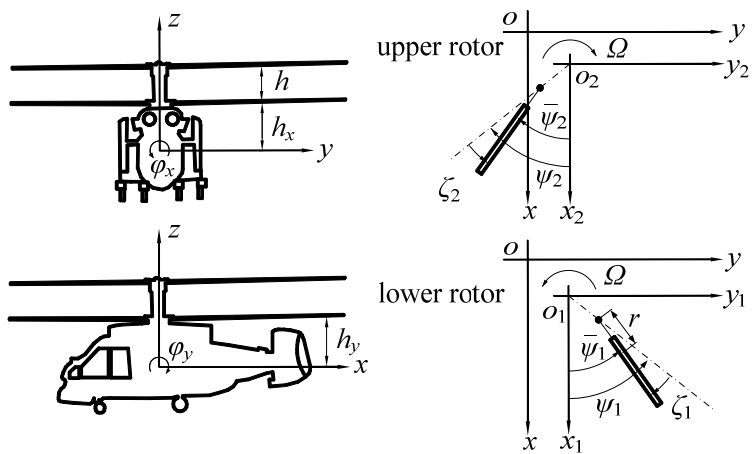

Figure 1.Analytical model of ground resonance for coaxial articulated rotor helicopter

Upper rotor and lower rotor are connected to the power transmission system via respective rotor shaft, and connected with body through the transmission system shell. Thus, there are coupling effects between upper rotor and lower rotor, as well as rotor and power/transmission system, from the torsional vibration system consisting of helicopter rotor/power/transmission. In the case of helicopter ground resonance, periodic lag motion (upper rotor and lower rotor) is coupled to motion of the centre of hub, however, there is no direct coupling between upper rotor and lower rotor.

According to Figure 1, considering small angle, the distance coordinate between blade of lower rotor and profile of lag hinge $r$ in the fixed coordinate system is:

$$
\left\{\begin{array}{l}
x_{1}=e \cos \psi_{1}+r \cos \bar{\psi}_{1}+h_{y} \varphi_{y} \\
y_{1}=e \sin \psi_{1}+r \sin \bar{\psi}_{1}-h_{x} \varphi_{x}
\end{array}\right.
$$

Where: $\bar{\psi}_{1}=\psi_{1}-\zeta_{1}$

Assuming that elastic deformation of upper rotor shaft is not taken into account, the coordinate of arbitrary profile of upper rotor blade is:

$$
\left\{\begin{array}{l}
x_{2}=e \cos \psi_{2}+r \cos \bar{\psi}_{2}+\left(h+h_{y}\right) \varphi_{y} \\
y_{2}=-e \sin \psi_{2}-r \sin \bar{\psi}_{2}-\left(h+h_{x}\right) \varphi_{x}
\end{array}\right.
$$

Where: $\bar{\psi}_{2}=\psi_{2}-\zeta_{2}$.

Finding the first and second derivative of time about formula (1) and (2), and obtaining speed and acceleration of blade arbitrary profile. Supposing lag stiffness of upper rotor and lower rotor blade is $K_{\zeta}$, and structural damping is $C_{\zeta}$, then lag motion equation of the $k$-th blade of lower rotor(for the sake of simplicity, the subscript $k$ is omitted) is:

$$
\begin{aligned}
& \int_{0}^{R-e} \bar{m} \ddot{x}_{1} \sin \bar{\psi}_{1} r \mathrm{~d} r-\int_{0}^{R-e} \bar{m} \ddot{y}_{1} \cos \bar{\psi}_{1} r \mathrm{~d} r \\
& +C_{\zeta} \dot{\zeta}_{1}+K_{\zeta} \zeta_{1}=0
\end{aligned}
$$

Equation of upper rotor blade lag motion is:

$$
\begin{aligned}
& \int_{0}^{R-e} \bar{m} \ddot{x}_{2} \sin \bar{\psi}_{2} r \mathrm{~d} r \\
& +\int_{0}^{R-e} \bar{m}_{2} \cos \bar{\psi}_{2} r \mathrm{~d} r+C_{\zeta} \dot{\zeta}_{2}+K_{\zeta} \zeta_{2}=0
\end{aligned}
$$

Where: $\bar{m}$ is density of blade mass; $R$ is rotor radius. Considering small lag angle, firstly, expanding the formula (3) and (4), and then obtaining disturbance equation of $k$-th blade lag motion(upper rotor and lower rotor) after omitting the small above second order and second order, The disturbance equations of each blade lag motion can be converted to the disturbance equations of rotor periodic lag motion by using multi-blade coordinate transformation. For ground or hovering helicopter, if number of rotor blades are three or more, periodic coefficient with time caused by azimuth angle of blade can be eliminated after multi-blade coordinate transformation, and the disturbance equation of constant coefficient of rotor periodic lag motion is obtained. In this way, the disturbance equations of lag motion of lower rotor after multi-blade coordinate transformation are:

$$
\begin{aligned}
& I_{b} \ddot{\zeta}_{1 \mathrm{~s}}+S_{b} h_{y} \ddot{\varphi}_{y}+C_{\zeta} \dot{\zeta}_{1 \mathrm{~s}}-2 \Omega I_{b} \dot{\zeta}_{1 \mathrm{c}} \\
& +\left[K_{\zeta}+\Omega^{2}\left(e S_{b}-I_{b}\right)\right] \zeta_{1 \mathrm{~s}}-\Omega C_{\zeta} \zeta_{1 \mathrm{c}}=0 \\
& I_{b} \ddot{\zeta}_{1 \mathrm{c}}+S_{b} h_{x} \ddot{\varphi}_{x}+C_{\zeta} \dot{\zeta}_{1 \mathrm{c}}+2 \Omega I_{b} \dot{\zeta}_{1 \mathrm{~s}} \\
& +\left[K_{\zeta}+\Omega^{2}\left(e S_{b}-I_{b}\right)\right] \zeta_{1 \mathrm{c}}+\Omega C_{\zeta} \zeta_{1 \mathrm{~s}}=0
\end{aligned}
$$

The disturbance equations of lag motion of upper rotor after multi-blade coordinate transformation are:

$$
\begin{gathered}
I_{b} \ddot{\zeta}_{2 \mathrm{~s}}+S_{b}\left(h+h_{y}\right) \ddot{\varphi}_{y}+C_{\zeta} \dot{\zeta}_{2 \mathrm{~s}}-2 \Omega I_{b} \dot{\zeta}_{2 \mathrm{c}} \\
+\left[K_{\zeta}+\Omega^{2}\left(e S_{b}-I_{b}\right)\right] \zeta_{2 \mathrm{~s}}-\Omega C_{\zeta} \zeta_{2 \mathrm{c}}=0 \\
I_{b} \ddot{\zeta}_{2 \mathrm{c}}-S_{b}\left(h+h_{x}\right) \ddot{\varphi}_{x}+C_{\zeta} \dot{\zeta}_{2 \mathrm{c}}+2 \Omega I_{b} \dot{\zeta}_{2 \mathrm{~s}} \\
+\left[K_{\zeta}+\Omega^{2}\left(e S_{b}-I_{b}\right)\right] \zeta_{2 \mathrm{c}}+\Omega C_{\zeta} \zeta_{2 \mathrm{~s}}=0
\end{gathered}
$$

Where: cosinoidal and sinusoidal components of periodic lag angle of lower rotor are $\zeta_{1 \mathrm{c}}$ and $\zeta_{1 \mathrm{~s}}$; cosinoidal and sinusoidal components of periodic lag angle of upper rotor are $\zeta_{2 \mathrm{c}}$ and $\zeta_{2 \mathrm{~s}}$; blade moment of inertia around lag hinge is $I_{b}$; blade static moment around lag hinge is $S_{b}$.

Setting linear damping and stiffness of body around the $x$-axis are $C_{x}$ and $K_{x}$, respectively, and damping and stiffness around the $y$-axis are $C_{y}$ and $K_{y}$, thus, the motion equations of body pitch and roll are:

$$
\begin{aligned}
& \quad \sum_{0}^{N} \int_{0}^{R-e} h_{x} \ddot{y}_{1} \bar{m} \mathrm{~d} r+\sum^{N} \int_{0}^{R-e}\left(h+h_{x}\right) \ddot{y}_{2} \bar{m} \mathrm{~d} r \\
= & I_{x} \ddot{\varphi}_{x}+C_{x} \dot{\varphi}_{x}+K_{x} \varphi_{x} \\
- & \left(\sum \int_{0}^{N-e} h_{y} \ddot{x}_{1} \bar{m} \mathrm{~d} r+\sum^{N} \int_{0}^{R-e}\left(h+h_{y}\right) \ddot{x}_{2} \bar{m} \mathrm{~d} r\right) \\
= & I_{y} \ddot{\varphi}_{y}+C_{y} \dot{\varphi}_{y}+K_{y} \varphi_{y}
\end{aligned}
$$

The motional equations after expanding and multiblade coordinate transformation are: 


$$
\begin{aligned}
& \frac{1}{2} N S_{b} h_{x} \ddot{\zeta}_{1 \mathrm{c}}-\frac{1}{2} N S_{b}\left(h+h_{x}\right) \ddot{\zeta}_{2 \mathrm{c}} \\
& +I_{x} \ddot{\varphi}_{x}+C_{x} \dot{\varphi}_{x}+K_{x} \varphi_{x}=0 \\
& \frac{1}{2} N S_{b} h_{y} \ddot{\zeta}_{1 \mathrm{~s}}+\frac{1}{2} N S_{b}\left(h+h_{y}\right) \ddot{\zeta}_{2 \mathrm{~s}} \\
& +I_{y} \ddot{\varphi}_{y}+C_{y} \dot{\varphi}_{y}+K_{y} \varphi_{y}=0
\end{aligned}
$$

Where: the body inertial moment around the $x$-axis and $y$-axis are $I_{x} 、 I_{y}$; fuselageinertialmoment around the $\mathrm{x}$-axis and y-axis are $I_{f x}, I_{f y}$.

The formula (5) (8) are lag equations of motions of upper rotor and lower rotor, formula(11) and (12) are equations of body motions, and they combine to form the coupling equations of motions between rotor and fuselage of coaxial helicopter

Setting $\mathbf{q}=\left[\begin{array}{llllll}\zeta_{1 \mathrm{~s}} & \zeta_{1 \mathrm{c}} & \zeta_{2 \mathrm{~s}} & \zeta_{2 \mathrm{c}} & \varphi_{x} & \varphi_{y}\end{array}\right]^{\mathrm{T}}$, the systematic equations of motions can be written in the form of matrix:

$$
\mathbf{M} \ddot{\mathbf{q}}+\mathbf{C} \dot{\mathbf{q}}+\mathbf{K q}=\mathbf{0}(13)
$$

Where: systematic mass matrix, damping matrix and stiffness matrix are $\mathbf{M} 、 \mathbf{C}$ and $\mathbf{K}$.

Then, ordering $\mathbf{Q}=\left[\begin{array}{ll}\dot{\mathbf{q}} & \mathbf{q}\end{array}\right]^{\mathrm{T}}$, the systematic equations of motions can be rewritten as the equations of state:

$$
\text { Where: the state matrix is } \mathbf{A}=\left[\begin{array}{cc}
-\mathbf{M}^{-1} \mathbf{C} & -\mathbf{M}^{-1} \mathbf{K} \\
\mathbf{I} & \mathbf{0}
\end{array}\right] \text {. }
$$

For formula (14), the characteristic analysis can be carried out, and initial disturbance response can also be calculated, if two methods can be combined, it will show a more complete systematic dynamics characteristic.

\section{Analytical method}

\subsection{Simulation parameters}

In the case of an articulated rotor, the parameters used are similar to the model used by the NASA Ames Research Centre $^{[13]}$, except for type of rotor, blade, and body structure damping, as shown in Table 1.

Table 1 Parameters of rotor and body

\begin{tabular}{lcc}
\hline Parameter & Unit & Value \\
\hline Number of rotor blade & $\mathrm{m}$ & 3 \\
Rotor radius & $\mathrm{m}$ & 0.8108 \\
Bladechord & $\mathrm{m}$ & 0.0419 \\
Offset of lag hinge & $\mathrm{kg} \cdot \mathrm{m}^{2}$ & 0.0173 \\
Inertia moment of blade about hinge & $\mathrm{m}$ & 0.2429 \\
Distance of blade centre to hub centre & $\mathrm{kg}$ & 0.2432 \\
Mass of blade (outside lag hinge) & $\%$ & 10.4 \\
Damping ratio of lag & $\mathrm{m}$ & 0.2405 \\
Distance of body rotation centre to lower & $\mathrm{m}$ & 0.2405 \\
Distance of upper rotor to lower rotor & $\mathrm{kg} \cdot \mathrm{m}^{2}$ & 0.607 \\
Inertia moment of pitch rotation & $\mathrm{Hz}$ & 1.59 \\
Natural frequency of pitch rotation & $\mathrm{kg} \cdot \mathrm{m} / \mathrm{rad}$ & 6.186 \\
Spring constant of pitch rotation & $\%$ & 32.0 \\
Damping ratio of pitch rotation & $\mathrm{kg} \cdot \mathrm{m}^{2}$ & 0.177 \\
Inertia moment of roll rotation & $\mathrm{Hz}$ & 3.96 \\
Natural frequency of roll rotation & $\mathrm{kg} \cdot \mathrm{m} / \mathrm{rad}$ & 11.201 \\
Spring constant of roll rotation & $\%$ & 18.58 \\
Damping ratio of roll rotation & &
\end{tabular}

\subsection{The results of eigenvalue and analysis}

According to the calculation results of eigenvalues, modal frequency and modal damping are plotted along with rotor speed. The results of the basic model are shown in Figure 2(a) and Figure 2(b). LA1 and LA2 express advance lag mode with significant lower rotor and upper rotor characteristic respective, and LR1 and LR2 express regressive lag mode with significant lower rotor and upper rotor characteristics, BX and BY, respectively, show body roll mode and body pitch mode. If the upper rotor turns positive, then in the fixed coordinate system, LA2 and LR2 modes follow the rotation of upper rotor, and their frequencies are positive Since the rotary direction of lower rotor is opposite to that of upper rotor, therefore, in the fixed coordinate system, the rotary directions of LA1 and LR1 modes should be the same with lower rotor, and their frequencies in the fixed coordinate system should be negative. However, in order to facilitate the comparison of modal frequencies, and the frequencies of LA1 and LR1 modes are displayed as positive.

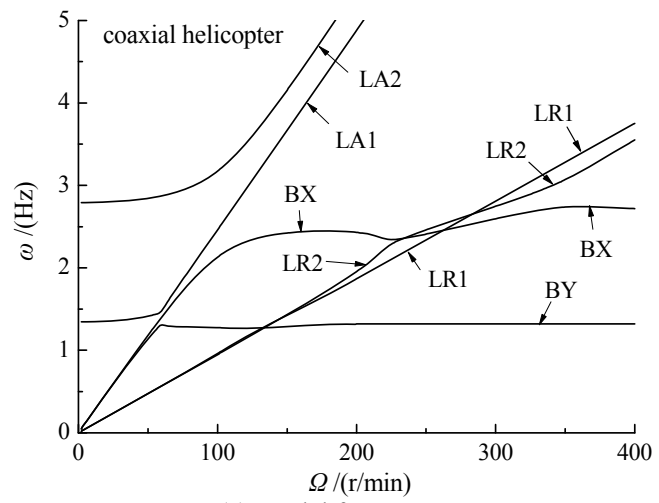

(a) Modal frequency

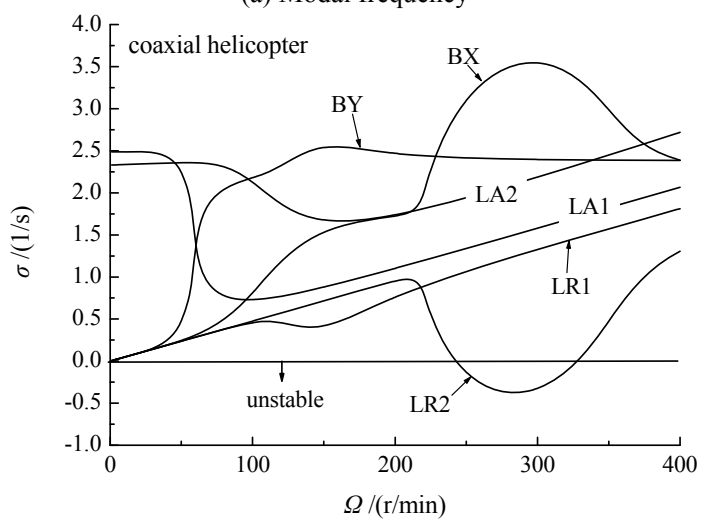

(b) Modal damping

Figure 2.Modal frequency / damping vs rotor speed

As can be seen from Figure 2(a), LA2、LR2 modes and body roll mode have strong coupling. LR2 mode is mainly regressive lag mode of upper rotor, and fundamental lag natural frequency of modal rotor blades is: $\omega_{\zeta}=\left(e S_{b} / I_{b}\right)^{1 / 2} \Omega=0.4345 \Omega$, The base frequency of isolated rotor regressive lag mode is: $\omega_{\mathrm{LR}}=\Omega$ $\omega_{\zeta}=0.5655 \Omega$ (upper rotor and lower are same), Therefore, its rotary direction is the same as that of upper rotor (clockwise) and is coupled to body roll mode of advance rotation (clockwise) in the fixed coordinate system. LR1 
mode performs regressive lag mode characteristic with upper rotor, its rotary direction is the same as that of lower rotor (counterclockwise), and LR1 mode is not coupled to fuselage mode of advance rotation. From modal frequency curves in Figure 2(a), it is found that modal frequency changes almost linearly along with rotor speed. When rotor speed is lower than unstable center (near $280 \mathrm{r} / \mathrm{min}$ ), LR2 modal frequency is higher than LR1 modal frequency. otherwise, they are just opposite. It shows that body roll motion provides a different "spring" effect on LR2 mode in the coupling region of body roll mode. The frequency of the body roll mode also corresponding changes in the coupling region, which indicates that periodic lag motion of upper rotor provides different "spring" effect on body roll mode.

From modal damping curves in Figure 2(b), modal damping of LR2 is greatly reduced in the speed region where regressive lag mode is coupled with body roll mode, and modal damping is less than zero in a certain rotary speed zone, however, body roll modal damping is improved greatly. LR1 modal damping is almost unaffected by body, indicating that there is no coupling between LR1 mode and fuselage. Therefore, LR2 mode is coupled with body roll mode, and leading to lower LR2 modal damping, it is the main reason to produce coaxial helicopter ground resonance.

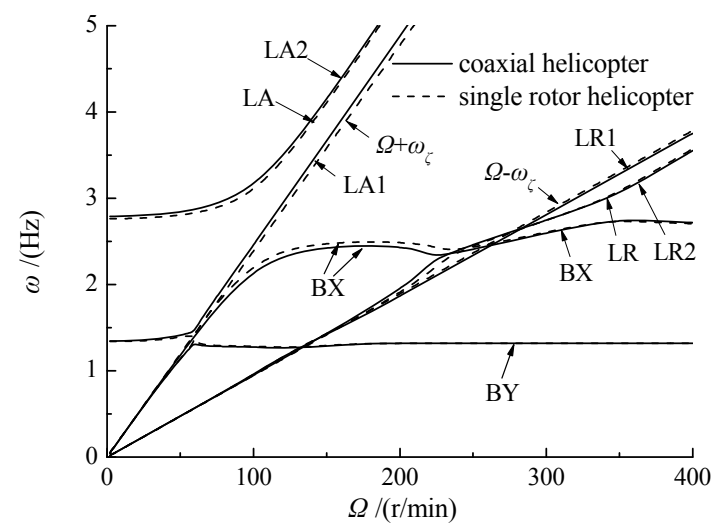

(a) Modal frequency

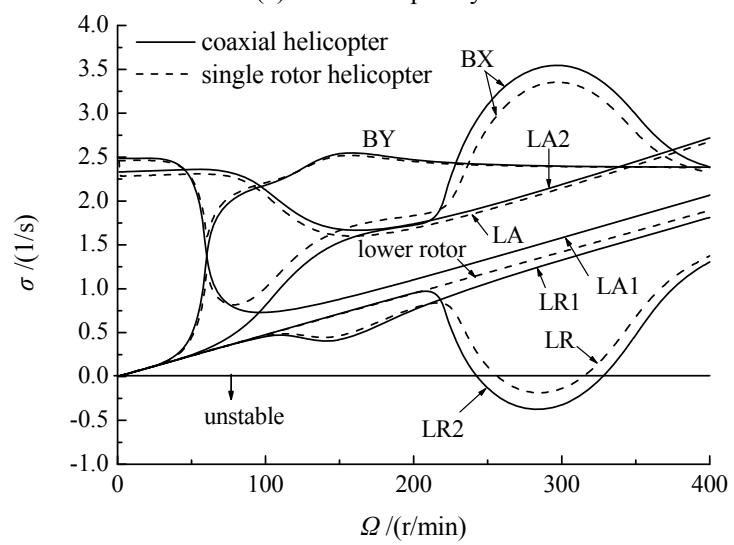

(b) Modal damping

Figure 3.Comparison of helicopter ground resonance modal characters between coaxial rotor and single rotor (body inertia moment is constant)

In order to understand the effect of lower rotor on coaxial helicopter ground resonance, lower rotor is retained, but inertial coupling between rotor and fuselage is removed, that is, inertial coupling terms of motional equations of lower rotor lag and body pitch and body roll are removed, and obtaining motional equations of single rotor helicopter ground resonance with only upper rotor and fuselage coupled, but body inertial moment remains unchanging, so the virtual configuration of single rotor helicopter and coaxial helicopter can be compared. modal frequency and damping curves of the single rotor helicopter ground resonance are shown in Figure 3(a), Figure 3(b). In order to facilitate comparison, curves of coaxial helicopter ground resonance are also shown in the same Figures. In the figure, solid lines indicate coaxial helicopter, and broken lines indicate single rotor helicopter.

Due to the removal of inertial coupling effect between lower rotor and fuselage, in fact, lower rotor behaves as lag characteristic of isolated rotor, from frequency curves in Figure 3(a), it is found that lag frequency of coaxial helicopter lower rotor is very close to it. It can also be seen from the curves that coupling effect of coaxial helicopter LR2 mode and body roll mode is stronger than that of single rotor helicopter. it is found that LR2 modal damping of coaxial helicopter is significantly lower than that of single rotor helicopter from modal damping curves in Figure 3(b). So it is shown that lower rotor enhance ground resonance instability of coaxial helicopter.

\subsection{Rotors-fuselage interaction analysis}

Numerical integration of disturbance equations of motions are used to obtain the change of response of various DOFs along with time. Taking initial angle as $\zeta_{1 \mathrm{~s}}(0)=\zeta_{1 \mathrm{c}}(0)=\zeta_{2 \mathrm{~s}}(0)=\zeta_{2 \mathrm{c}}(0)=0.01, \quad \varphi_{x}(0)=\varphi_{y}(0)=0, \quad$ and initial angular velocity is zero, rotor speed is $\Omega=280$ $\mathrm{r} / \mathrm{min}$. From curves in Figure 3, it is found that LR2 modal damping is less than zero and other modal damping is greater than 1.0. Therefore, except for LR2 mode, other modes are attenuated in a very short time in the time course of response of various DOFs. In order to minimize impact of other modes, it needs to take response after a sufficiently long integration time for analysis. it shows response curves of coaxial helicopter various DOFs in Figure 4(a), (b), And Figure 5 shows response curves of single rotor helicopter various DOFs.

Comparing the response of coaxial helicopter various DOFs and single rotor helicopter various DOFs, what we can see is that phase relationship between periodic lag motion of upper rotor and body roll motion of coaxial helicopter is almost the same with that of single rotor helicopter, but in the same initial conditions, its response is much greater than that of single rotor helicopter after the same 8 second, it is Indicated that LR2 modal damping of coaxial helicopter is less than LR modal damping of single rotor helicopter. And amplitude of periodic lag response of coaxial helicopter lower rotor is about half that of upper rotor.

We can analyze phase relationship of various DOFs from the response curves. For LR2 mode in the fixed coordinate system, the periodic lag vector will be rotated at the frequency of LR2 mode by following rotary direction of upper rotor (or single rotor), and upper 
rotor(or single rotor) lag sinusoidal component $\zeta_{2 \mathrm{~s}}\left(\right.$ or $\left.\zeta_{\mathrm{s}}\right)$ and body roll vector $\varphi_{x}$ are close to opposite phase(for an undamping system, they are just in opposite phase), while cosinoidal component $\zeta_{2 \mathrm{c}}\left(\right.$ or $\zeta_{\mathrm{c}}$ ) is ahead of sinusoidal component about $90^{\circ}$, and the corresponding angular velocity vector is ahead of angular displacement vector about $90^{\circ}$; On the other hand, upper rotor lag sinusoidal component $\zeta_{1 \mathrm{~s}}$ and body roll vector $\varphi_{x}$ are close to same phase(for an undamping system, they are just in same phase), while cosinoidal component $\zeta_{1 \mathrm{c}}$ is ahead of sinusoidal component about $90^{\circ}$, also, and angular velocity vector is ahead of angle displacement vector about $90^{\circ}$, It is shown that both rotor periodic lag motion and body roll motion are rotated with LR2 modal frequency $\omega_{\text {LR2 }}$ following rotary direction of upper rotor. it is found that body roll motion and body pitch motion with are same phase from curves in Figure 4, Figure 5, but amplitude of roll motion is much larger, which is consistent with phenomenon of helicopter ground resonance when body is anisotropy. In order to facilitate analysis, interaction between body pitch motion and rotor is not considered, and interaction between body roll motion and rotor periodic lag is mainly analyzed. The phase relationships of various DOFs of coaxial and single rotor helicopter are shown in Figure 6(a) and Figure 6(b), respectively.

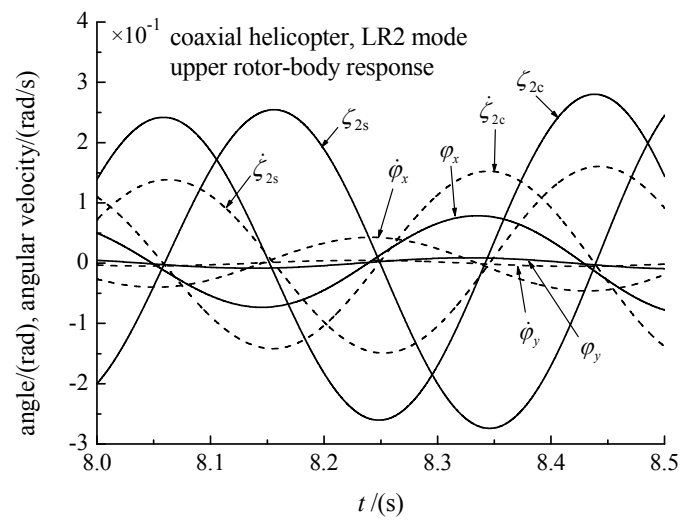

(a) Upper rotor and body

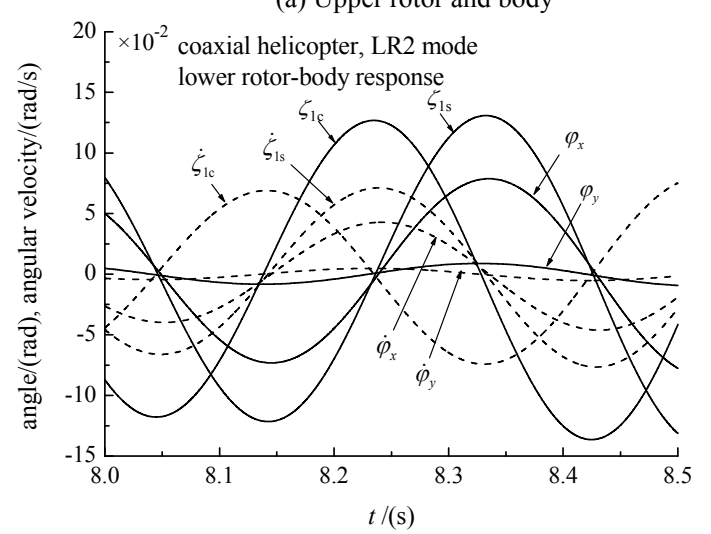

(b) Lower rotor and body

Figure 4.Response of various DOFs(Coaxial rotor helicopter, LR2 Mode, $\Omega=280 \mathrm{r} / \mathrm{min}$ )

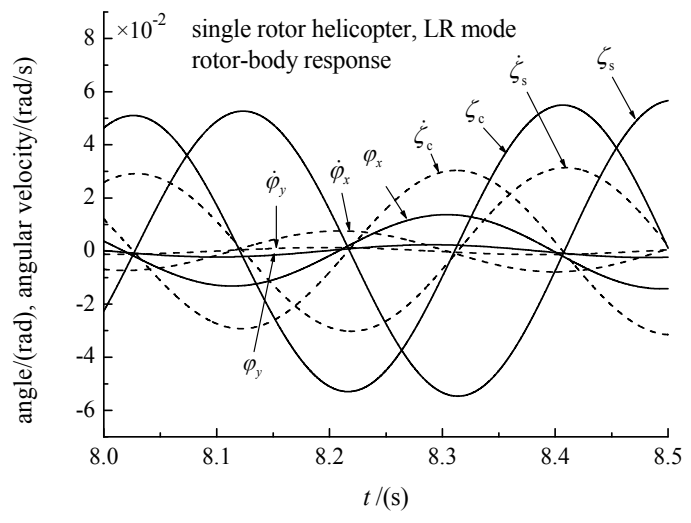

Figure 5.Response of various DOFs(Single rotor helicopter, LR mode, $\Omega=280 \mathrm{r} / \mathrm{min}$ )

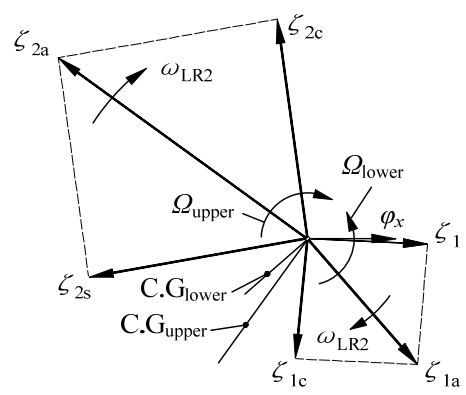

(a)Coaxial helicopter, LR2 mode

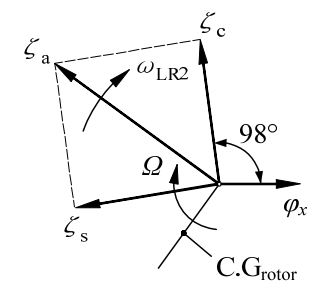

(b)Single rotor helicopter, LR mode

Figure6.Phase relation of various $\operatorname{DOFs}(\Omega=280 \mathrm{r} / \mathrm{min})$

Firstly, interaction between upper rotor(or single rotor) periodic lag and body roll motion of coaxial helicopter is analyzed.

Then, we can see from equation (11), for this upper rotor, there is only lag cosinoidal periodic component has inertia moment effect on body roll motion, Moment of inertia is: $M_{\mathrm{RB} 2}=-N S_{b}\left(h+h_{x}\right) \ddot{\zeta}_{2 \mathrm{c}} / 2$, Thus, when $\ddot{\zeta}_{2 \mathrm{c}}$ and $\dot{\varphi}_{x}$ are in the same phase, the symbol of $M_{\mathrm{RB} 2}$ and body roll damping moment is opposite. However, as can be seen from Figure 6 , When $\ddot{\zeta}_{2 \mathrm{c}}\left(\right.$ or $\left.\ddot{\zeta}_{\mathrm{c}}\right)$ and $\zeta_{\mathrm{c} 2}\left(\right.$ or $\left.\zeta_{\mathrm{c}}\right)$ are in the opposite phase, and be ahead of $\varphi_{x}$ vector approaches to $90^{\circ}$. That is, when $\ddot{\zeta}_{2 \mathrm{c}}$ (or $\ddot{\zeta}_{\mathrm{c}}$ ) and $\dot{\varphi}_{x}$ are close to the same phase, it provides a negative damping to body, namely, inputting energy to body.

Because of center of rotor gravity lags lag vector (Figure 6) $90^{\circ}$, and moment of body inertia and vector $\varphi_{x}$ is in the opposite phase, body provides excitation to periodic lag motion of upper rotor, namely, inputting energy to lag of rotors. Lag sinusoidal component $\zeta_{2 \mathrm{~s}}$ (or $\zeta_{\mathrm{s}}$ ) of upper rotor and body roll angle $\varphi_{x}$ are close to opposite phase, so there is almost no energy transferred between $\varphi_{x}$ and $\zeta_{2 \mathrm{~s}}\left(\right.$ or $\zeta_{\mathrm{s}}$ ), and they are mainly provided 
positive or negative "spring" constraint. Thus, for this LR2 mode (or single rotor helicopter LR mode), instability of helicopter ground resonance is mainly due to energy transferred between periodic lag motion of upper rotor and body roll rotation.

Next, interaction between lower rotor and body can also be analyzed combining with equation (11) according to phase relationships as shown in Figure 6(a). According to equation (11), for this lower rotor, there is only lag cosinoidal periodic motion has inertia moment effect on body roll motion, too, the size is $M_{\mathrm{RB} 1}=N S_{b} h_{x} \ddot{\zeta}_{1 c} / 2$, when $\ddot{\zeta}_{1 c}$ and $\dot{\varphi}_{x}$ are in the opposite phase, lower rotor provides negative damping to body; As rotary direction of lower rotor is opposite to that of upper rotor, and center of rotor gravity lags lag vector (shown in Figure 6) $90^{\circ}$, Therefore, moment body inertia has an incentive effect on periodic lag motion of upper rotor, that is, body transfers energy to rotors. In summary, for this LR2 mode, instability of helicopter ground resonance is mainly due to energy transferred between periodic lag motion of upper rotor and body roll rotation. For this stable region (such as $\Omega=350 \mathrm{r} / \mathrm{min}$ ), the response and phase relation of rotors and fuselage are shown in Figure 7 and Figure 8. It can be found that upper rotor and lower rotor center of gravity and body roll vector is almost in the opposite phase, periodic lag motion and body roll will not transfer energy to each other.

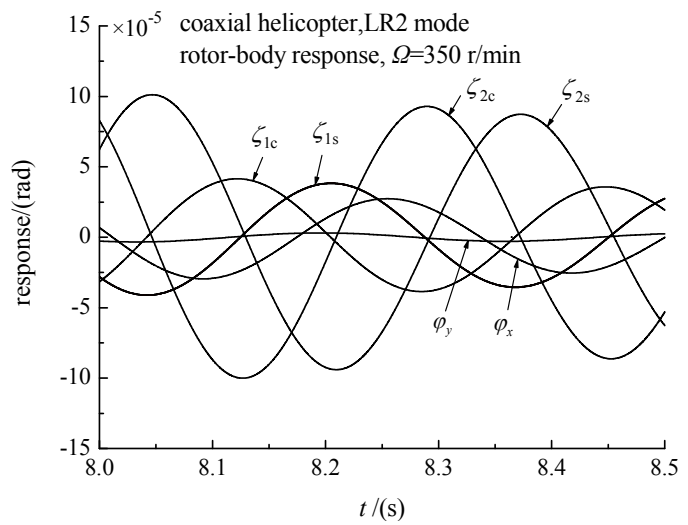

Figure 7.Response of various DOFs(LR2mode, $\Omega=350 \mathrm{r} / \mathrm{min}$ )

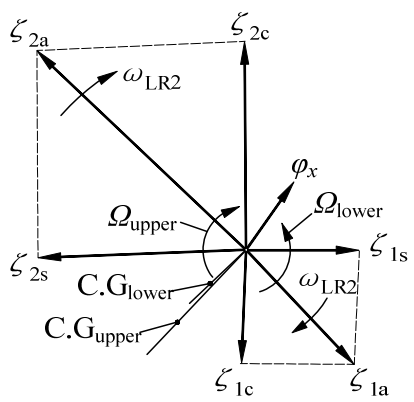

Figure 8.Phase relation of various DOFs(LR2mode, $\Omega=350 \mathrm{r} / \mathrm{min}$ )

For the situation of body is isotropic(body pitch parameters are consistent with that of body roll ), modal frequency and modal damping of system are quite different from those of the situation of body is anisotropy (Figure 9(a) and (b)), Providing that upper rotor turns positive, then, regressive lag LR2 mode with upper rotor characteristic and body mode of forward rotation B2 are coupled, and the modal damping is significantly lower than LR2 modal damping when body is anisotropy, it shows that dynamic instability of helicopter ground resonance is higher when body is isotropic, which is consistent with conclusion of analysis for single rotor helicopter ground resonance ${ }^{[1,2]}$.

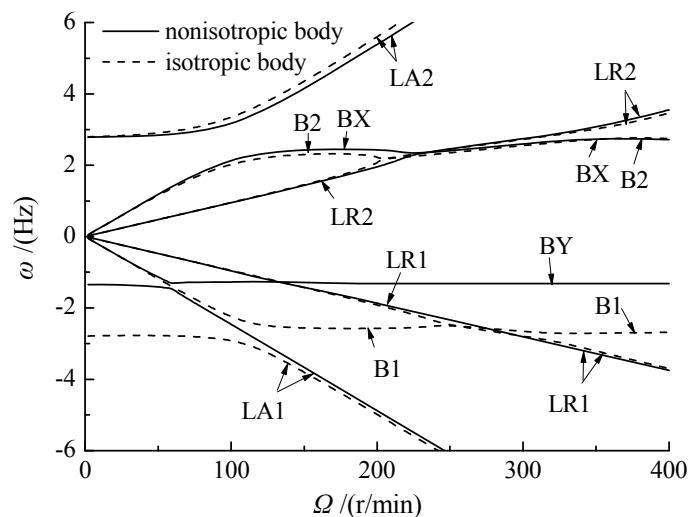

(a) Modal frequency

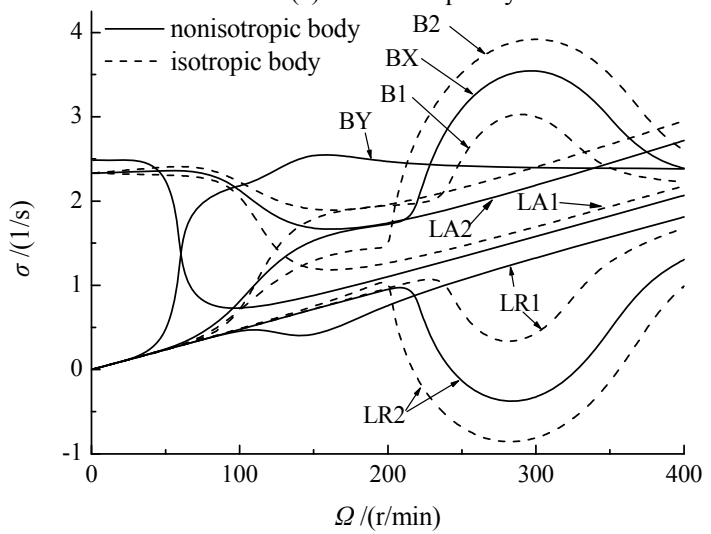

(b) Modal damping

Figure 9.Comparison of helicopter ground resonance modal characters between isotropic and nonisotropic body

Since pitch characteristic of body is in accordance with roll characteristic of body, frequency of body B1 mode is higher than frequency of pitch model (BY) when body is anisotropy, however, its rotary direction is the same as that of lower rotor, and it coupled with regressive lag LR2 mode with lower rotor characteristic, so that LR1 modal damping is significantly lower than damping when body is anisotropy, but is much higher than LR2 modal damping. Whether body is anisotropy or isotropic, there is both forward and reverse rotation of body mode of coaxial helicopter, however, there is only forward rotation when body of single rotor helicopter is isotropic. When body is isotropic, LR2 modal damping is significantly lower than damping value when body is anisotropic, which can be explained based on its response characteristic and phase relationship. On the other hand, when body is isotropic, for this unstable region $(\Omega=280 \mathrm{r} / \mathrm{min})$, periodic lag motion of rotor and response process of body are shown in Figure 10, phase relationship of various DOFs are shown in Figure 11 (the amplitude of periodic lag motion of lower rotor is very small and is not shown). 


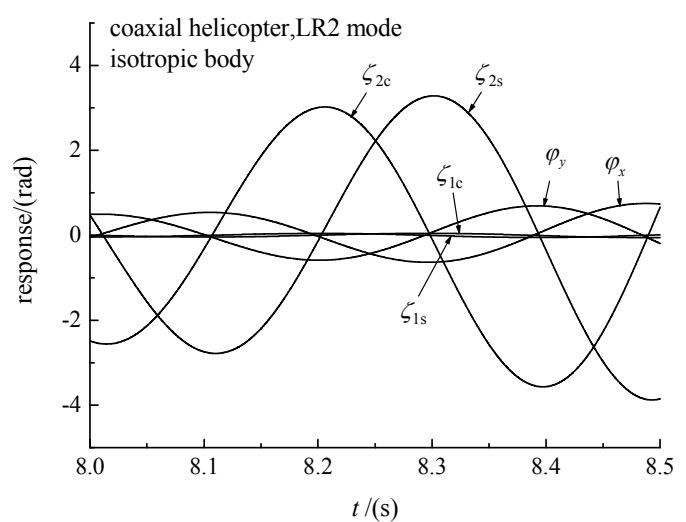

Figure 10.Response of various DOF(mode LR2, isotropy)

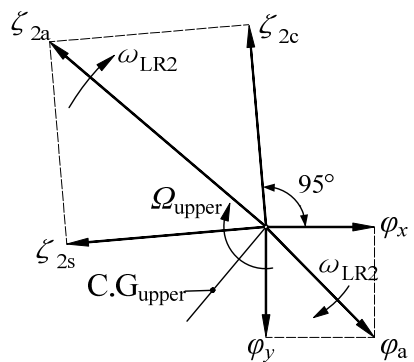

Figure 11.Phase relation of various DOF(mode LR2, isotropy)

As can be seen from Figure 10 and 11, when body is isotropic, for LR2 mode, periodic lag motion of upper rotor and body roll motion and pitch motion form a relationship of energy transferred, when body is anisotropy, energy transferred between rotor and body is increased, thus, increasing instability of helicopter ground resonance. While the amplitude of periodic lag motion of lower rotor is relatively small and almost be ignored, and this feature is obviously different from the situation when body is anisotropic.

As for the instability of coaxial helicopter ground resonance, it is also need to observe that the size of energy transferred between rotor and body and energy of structural damping consumes. Taking lag of lower rotor and upper rotor and body roll motion as the object of study as before, because one DOF provide negative damping to another DOF, in fact, it is doing positive power, so upper rotor and lower rotor power on body $W_{\mathrm{RB} 2}$ and $W_{\mathrm{RB} 1}$ are:

$$
\begin{array}{r}
W_{\mathrm{RB} 2}=-\int_{t} M_{\mathrm{RB} 2} \cdot \dot{\varphi}_{x} \mathrm{~d} t \\
=\frac{1}{2} N S_{b}\left(h+h_{x}\right) \int_{t} \ddot{\zeta}_{2 \mathrm{c}} \dot{\varphi}_{x} \mathrm{~d} t \\
W_{\mathrm{RB} 1}=-\int_{t} M_{\mathrm{RB} 1} \cdot \dot{\varphi}_{x} \mathrm{~d} t \\
=-\frac{1}{2} N S_{b} h_{x} \int_{t} \ddot{\zeta}_{1 \mathrm{c}} \dot{\varphi}_{x} \mathrm{~d} t
\end{array}
$$

And body powers on upper rotor and lower rotor $W_{\mathrm{BR} 2}$ and $W_{\mathrm{BR} 1}$ are:

$$
W_{\mathrm{BR} 2}=-\int_{t} M_{\mathrm{BR} 2} \cdot \dot{\zeta}_{2 \mathrm{c}} \mathrm{d} t=S_{b}\left(h+h_{x}\right) \int_{t} \ddot{\varphi}_{x} \dot{\zeta}_{2 \mathrm{c}} \mathrm{d} t
$$

$$
W_{\mathrm{BR} 1}=-\int_{t} M_{\mathrm{BR} 1} \cdot \dot{\zeta}_{\mathrm{lc}} \mathrm{d} t=-S_{b} h_{x} \int_{t} \ddot{\varphi}_{x} \dot{\zeta}_{\mathrm{lc}} \mathrm{d} t
$$

Using $\Delta W_{1}$ to show the sum of power transferred between lower rotor and body, this is $\Delta W_{1}=\Delta W_{\mathrm{RB} 1}+\Delta W_{\mathrm{BR} 1}$, and using $\Delta W_{2}$ to show the sum of power transferred between lower rotor and body, this is $\Delta W_{2}=\Delta W_{\mathrm{RB} 2}+\Delta W_{\mathrm{BR} 2}$. In unstable region of LR2 mode, energy transferred between periodic lag motion of rotors and body roll rotation was observed. In order to minimize the impact of other modes, starting to calculate energy transferred after 8 second, and the results show in Figure 12 and Figure 13.

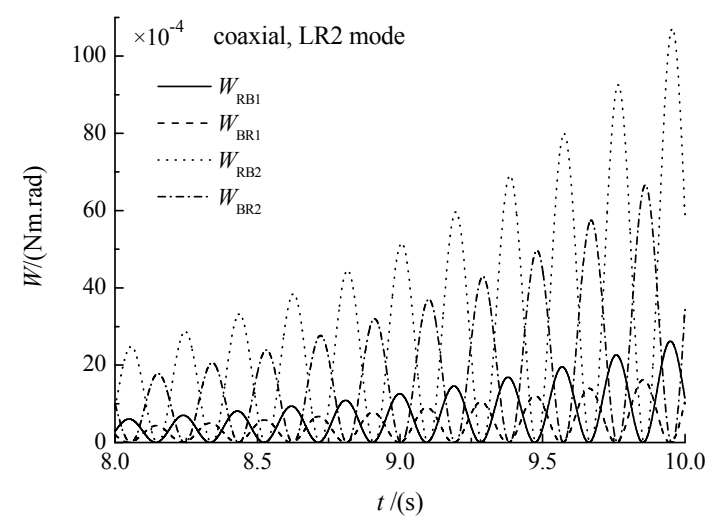

Figure12.Interaction history of rotors and body

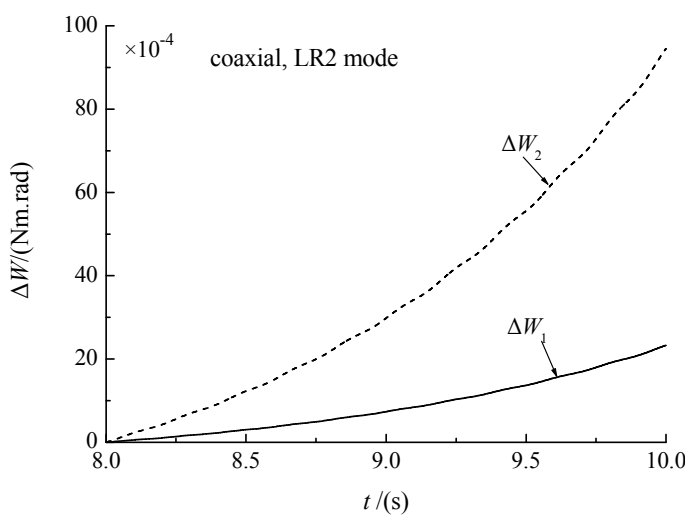

Figure 13.Increment of system power vs time

We can see from Figure 12, it is positive power transferred between periodic lag motion of upper rotor and lower rotor and body roll rotation, and it shows characteristic of alternating change with time, Its frequency is equal to that of LR2 mode, and body does not power on rotors when the maximum moment of rotors power on body, in addition, rotors do not power on body when the maximum moment of body powers on rotors, it shows accumulative power of coaxial helicopter rotor and body will increase with time in Figure 13, and the effect of upper rotor is significantly greater than that of lower rotor. Therefore, for LR2 mode, the main cause of coaxial helicopter ground resonance is interaction between periodic lag of upper rotor and body, while lower rotor enhances instability of coaxial helicopter ground resonance, this explains the intrinsic reason that dynamic instability of coaxial helicopter ground 
resonance is higher than that of single rotor helicopter. Of course, although the amplitude of body pitch motion is much smaller than that of body roll motion, considering interaction between body pitch motion and rotors, there maybe some change of value, but it will not affects the final results of analysis.

\section{Conclusion}

(1) A time-frequency analysis method is proposed to reveal investigation of coaxial articulated helicopter ground resonance with structural damping. For mode with the least damping, interaction between periodic lag of upper rotor and lower rotor and body motion is analyzed by applying response characteristics of timefrequency, and internal reason of dynamic instability of coaxial helicopter ground resonance is explained.

(2) When body of coaxial helicopter is anisotropic, regressive lag LR2 mode with upper rotor characteristic is coupled with body roll mode, and it arouses body roll motion with the same direction of upper rotor rotation. While regressive lag LR1 mode with upper rotor characteristic and LR2 is in the opposite direction of rotation, there is not coupling between LR1 and body roll motion of reverse rotation, thus, helicopter ground resonance is caused by the coupling of regressive lag LR1 mode and body roll motion.

(3) In unstable rotary speed region of LR2 mode, coaxial helicopter ground resonance is mainly due to energy transferred between periodic lag motion of upper rotor and body roll rotation; In addition, energy transferred between periodic lag motion of lower rotor and body roll rotation improve dynamic instability of ground resonance.

(4) When body is isotroypic, there are two body modes B1 and B2 of coaxial helicopter that their frequency are similar and rotate reversely, this is significant difference from single rotor helicopter with only one body mode. B1 is the same as rotation of lower rotor and coulped with LR1 mode, and B2 is the same as rotation of upper rotor and coulped with LR2 mode, damping of LR2 mode is significantly lower than damping value when the fuselage is anisotropic.

(5) When body is isotropic, there is energy transferred between periodic lag motion of upper rotor and body roll rotation in unstable rotary speed region of LR2 mode, and instability of coaxial helicopter ground resonance is improved obviously comparing with body is anisotropy. While the effect of lower rotor and body motion is almost ignored.

\section{References}

1. Coleman M L, Feingold A M, Theory of self-excited mechanical oscillation of helicopter rotor with hinged blades, NACA Report TR-1351 (1958)

2. Zhang X G, Physical understanding of helicopter air and ground resonance, J. AHS, 31,4,4-11(1986)

3. Ormiston, $\mathrm{R}$ A, Rotor-fuselage dynamics of helicopter air and ground resonance,J. AHS, 36, 2,320(1991)
4. Russell E, Jennie S, Apache Helicopter Stabilization Using Neural Dynamic Programming, J. Guidance, Control\&Dynamics, 25, 1, 19-25(2002)

5. W Yuan, J Katupitiya, A Two-Stage Method for the Parametric Identification of Scale-Model Helicopter Dynamics, Proceedings ofAIAA Atmospheric Flight Mechanics Conference, 4733(2012)

6. Giuseppe Q, Vincenzo M, Pierangelo M. Lead-Lag Damper Robustness Analysis for Helicopter Ground Resonance, J. Guidance, Control\&Dynamics, 36, 4, 1150-1161(2013)

7. Tang D M, Dowell E H, Influence of nonlinear blade damping on helicopter ground resonance stability, J. Aircraft, 23,2, 104-110(1986)

8. Smith E C, Govindswamy K, Beale M R, Aeroelastic response and stability of helicopters with elastomeric lag damper,J. AHS,43, 3, 257-266(1996)

9. Sahasrabudhe V, Gold P J. Reducing rotor-body coupling using active control, Proceedings of AHS 60th Annual Forum,1118-1834(2004)

10. Zhao Y S, Choi Y T, Wereley N M, Semi-active damping of ground resonance in helicopter using magnetorheological dampers, J. AHS, 49, 4, 468482(2004)

11. Bousman W G, An experimental investigation of the effects of aeroelastic couplings on aeromechanical stability of a hingeless rotor helicopter,J. AHS, 26, 1, 46-54(1981)

12. Liu L, Anand V R, Keith R H, Friedrich K S, Ground Resonance and Whirl Stability Analysis Using CAMRAD, Proceedings of AHS 70th Annual Forum,2586-2596(2014)

13. McNulty M I, Bousman W G, Integrated technology rotor methodology assessment workshop, NASA CP10007(1988)

14. Wang, J.M., Duh,J, Fuh,J, Stability of the Sikorsky S-76 bearingless main rotor, Proceedings of AHS 49th Annual Forum, 983-1009(1993)

15. Krysinski T, Ferullo D, Overview of the EC155 dynamics validation program from design stage up to certification, Proceedings of AHS 55th Annual Forum, 1056-1063(1999)

16. Narramore J M, Mithat Yuce, Bell 429 main rotor aerodynamic and dynamic development, Proceedings of AHS 66th Annual Forum,436452(2010)

17. Zhang X G, Liu Q, The application of complex coordinates and mutual excitation analysis to the investigation of helicopter ground resonance, Acta Aeronautica et Astronautica Sinica,13, 11, 586593(1992)

18. Lu M, Fei B J, Ren Z Y, A numerical analysis for the ground resonance of a two-blade helicopter rotor on anisotropic flexible supports, Acta Aeronautica et Astronautica Sinica, 16, 3, 348-354(1995) 